\title{
INVESTIGACIONES
}

\section{Evaluación de una Unidad Didáctica sobre la enseñanza y aprendizaje de los conceptos de perímetro y área}

\author{
Evaluation of a didactic unit on teaching and learning the perimeter and area concepts
}

\author{
Constanza Muñoz Moreno ${ }^{a}$, Nielka Rojas González ${ }^{b}$ \\ ${ }^{\text {a }}$ Universidad Católica del Norte, Chile \\ Correo electrónico: constanzarociomunozmoreno@gmail.com

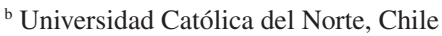 \\ Correo electrónico: nrojas03@ucn.cl
}

\section{RESUMEN}

Presentamos el análisis de una unidad didáctica cuyo objetivo es que los estudiantes construyan su propio significado de perímetro y área, y desarrollen la capacidad de resolver problemas mediante estrategias y tareas matemáticas contextualizadas, que presenten situaciones desafiantes que requieran poner en juego habilidades, destrezas y conocimientos, no necesariamente con la aplicación de fórmulas o algoritmos que conducen a los resultados de forma procedimental. La unidad didáctica se diseña siguiendo un marco específico del Análisis Didáctico que permite secuenciar las tareas en función a aspectos conceptuales y cognitivos, relativos a los temas de perímetro y área, luego se implementa en un nivel escolar de cuarto año de Educación Básica y se evalúan los resultados. El trabajo tiene como aporte central la confección de un material de enseñanza que vincula dos temas matemáticos, que tienden a enseñarse de forma aislada.

Palabras clave: Perímetro, Área, Educación Básica, Tareas matemáticas.

\begin{abstract}
This paper presents the analysis of a didactic unit designed with the purpose of guiding students toward building their own meaning of the mathematical concepts: perimeter and area. The didactic unit also aimed to develop students' ability to solve problems by presenting challenging situations through the use of strategies and contextualized mathematical tasks. These challenging situations engaged students to use their knowledge, skills, and abilities to get to results instead of applying procedural methods such as formulas or algorithms. The didactic unit was designed following the framework of Didactic Analysis, which allows the sequencing of tasks according to conceptual and cognitive aspects that are related to issues of perimeter and area. After its design, it was implemented in fourth grade primary students and the results of its implementation were evaluated. The main contribution of this work was the creation of teaching material that associates two mathematical topics that are usually taught in isolation.
\end{abstract}

Key words: Perimeter, Area, Primary Education, Mathematical tasks. 


\section{PLANTEAMIENTO DEL PROBLEMA}

Los docentes de Educación Básica de Matemática tienen un rol fundamental en el desarrollo integral de los estudiantes a través de la acción directa en el aula. Para ello se debe promover que los alumnos adquieran nuevos conocimientos y desarrollen habilidades que los lleven a una comprensión más profunda de la matemática. Para lograr este objetivo es necesario desarrollar metodologías de enseñanza significativas, generar situaciones de aprendizaje centrada en el significado de los conceptos matemáticos, realizar discusiones matemáticas en el aula, entre otros aspectos que lleven a estimular la curiosidad y comprensión de la matemática. Por ello, esta investigación surge de la necesidad de generar una unidad didáctica, específicamente en el eje temático de medición, ya que los contenidos establecidos por el currículo en este eje tienden a estudiarse a partir de algoritmos estandarizados o aplicación de fórmulas que les permite a los alumnos de manera procedimental llegar a los resultados (Espinoza, Barbé y Gálvez, 2011).

En el transcurso de la experiencia docente se ha identificado que los alumnos hacen uso incorrecto de algunos términos matemáticos o presentan razonamientos imprecisos cuando los utilizan. Específicamente, al estudiar las expresiones de perímetro y área se reducen a la expresión de una fórmula consensuada y determinada. Por ejemplo, Olmo, Moreno y Gil (1993) aseguran sobre dificultades y errores en el aprendizaje, que la confusión de perímetro y área es bastante frecuente, incluso el hecho de que dos figuras tengan la misma área induce a algunos estudiantes a pensar que tienen el mismo perímetro. En otros casos, calculan el área y el perímetro de una figura, y le asignan el valor mayor al área y el menor al perímetro. Asimismo, Corberán (1996) expresa que

esta falsa relación entre el área y el perímetro, que se ha constatado está muy arraigada en los alumnos, pone de manifiesto que estos piensan en el área y en el perímetro como en dos propiedades de la superficie íntimamente ligadas, concepción errónea que les impide ver el área como una propiedad de la superficie independiente del perímetro, que les dificulta e incluso imposibilita realizar transformaciones de superficies bajo determinadas condiciones (p.37).

En este sentido, uno de los grandes retos en Educación Matemática se relaciona con el análisis de los procesos de comprensión de conceptos matemáticos específicos por parte de los estudiantes. Por consiguiente, es necesario diseñar actividades contextualizadas que permitan a los alumnos comprender los conceptos de perímetro y área y la independencia entre ambos; así, se espera que puedan demostrar comprensión, usando diversos materiales y contextos, y que alcancen progresivamente un nivel de abstracción mayor.

En este contexto, surge la necesidad de crear una unidad didáctica, que aborde la transición del aprendizaje de los conceptos de perímetro y área. Tal como plantea Corberán (1996), para evitar estas dificultades se tienen que trabajar los conceptos de perímetro y área desde el inicio de la enseñanza. La unidad didáctica está centrada en el nivel de $4^{\circ}$ año de Educación Básica, ya que según el currículo nacional chileno el concepto de perímetro se estudia en $3^{\circ}$ año, mientras que el área comienza a trabajarse en $4^{\circ}$ año. Cabe destacar, que el material diseñado contempla tareas en las que se somete las superficies a determinadas transformaciones, que buscan relacionar las medidas de longitud y de área (medidas convencionales y no convencionales). Además, se presentan tareas en diversos 
contextos matemáticos, con distintas representaciones y que atiendan a los significados de los conceptos. De lo expuesto, se desprende el objetivo del estudio que es evaluar la unidad didáctica diseñada e implementada sobre la enseñanza y aprendizaje de los conceptos de perímetro y área en $4^{\circ}$ año de Educación Básica.

\section{MARCO TEÓRICO}

En Chile la Ley 20.370, más conocida como Ley General de Educación (LGE), en su principio de educación integral, basado en el desarrollo de múltiples formas de conocer, considera como relevante los aspectos físicos, sociales, morales, estéticos, creativos y espirituales, con atención especial a la integración de todas las ciencias, artes y saberes disciplinares. Por esto es necesario desarrollar estrategias de aprendizaje que aseguren que todos los estudiantes, independientemente de sus contextos, alcancen los objetivos generales y los estándares de aprendizaje, que contemplan estos destacados aspectos.

Del mismo modo, en el año 2012 el Ministerio de Educación chileno, en pos de satisfacer este afán, contribuyó entregando un currículo fundamentado en las Bases Curriculares para la Educación Básica, herramientas que guían el desarrollo de conocimientos, habilidades y actitudes de los estudiantes en todas las asignaturas. En este contexto, las Bases Curriculares conciben la asignatura de Educación Matemática como una herramienta que permite al alumno el desarrollo de un pensamiento lógico, metódico, crítico, autónomo, y de actitudes como la exactitud y el rigor. Por tanto, utilizamos el análisis didáctico en educación matemática, propuesto por Rico, Lupiáñez y Molina (2013), que surge de la acción investigadora y formadora de un amplio grupo de profesionales de la educación matemática que vienen empleando un mismo sistema de categorías y un conjunto de métodos para planificar la actividad matemática del profesor con el objeto de desarrollar variadas habilidades.

Esta investigación contempla el análisis didáctico como una estructura cíclica, en el cual distintas fases permiten diseñar la unidad didáctica (Rico, 2013; Rico, Lupiáñez y Molina, 2013). Para ello, realizamos un análisis focalizado en cinco análisis parciales que conforman el análisis didáctico, tales como el análisis conceptual, que lleva a profundizar en conceptos básicos de perímetro y área; el análisis curricular, que orienta los procesos de planificación de acuerdo con lo que establece el currículo nacional y en concordancia al nivel educativo. Por otro lado, efectuamos el análisis de contenido que lleva a generar la estructura y el análisis formal del tema de estudio, profundizando en los significados de los conceptos, aspectos fenomenológicos y los sistemas de representación que dan origen a los temas matemáticos. El tercer análisis es el cognitivo, que lleva a conocer las limitaciones, obstáculos, errores y dificultades que surgen en el proceso de enseñanza y aprendizaje de perímetro y área, aspectos que se contemplarán en el diseño de la unidad didáctica. Luego, se realiza el análisis de instrucción que permite secuenciar y definir las tareas matemáticas que conforman la unidad didáctica que se implementa en $4^{\circ}$ año de Educación Básica. Finalmente, el análisis evaluativo contribuye a la evaluación de la unidad didáctica, con la generación de conclusiones y síntesis respecto del proceso del diseño e implementación de ella, y con la toma de decisiones para las adaptaciones que puedan surgir a partir de este ciclo.

Desde el ámbito curricular, las Bases Curriculares chilenas (2012), formalmente indican que desde $3^{\circ}$ año de Educación Básica se han de trabajar medidas de longitud y 
superficie, aunque hemos de indicar que los estudiantes desde los primeros niveles escolares identifican y comparan longitudes de objetos usando palabras como largo y corto, y trabajan comparación de medidas de longitudes no estandarizadas. Asimismo, determinan longitudes de objetos usando distintas medidas (estandarizadas y no estandarizadas) en situaciones problemáticas. Se presentan una gradualidad de distintas habilidades que deben poner en práctica, como identificar, comparar y determinar longitudes, aspectos que les permite construir los conceptos de perímetro y área. La habilidad de medir longitudes y áreas se presenta en los niveles posteriores de $3^{\circ}$ a $5^{\circ}$ año de Educación Básica, y los contenidos se proyectan a $6^{\circ}$ año, como el cálculo de superficies de cubos y paralelepípedos.

Desde el ámbito matemático, la medición es una comparación con referentes fijos, que implica cuántas veces "algo" (una unidad) está contenida en el objeto a medir. Las unidades pueden ser de longitud de área o de volumen. En este trabajo atendemos a las dos primeras, por ser la temática que se abordan en $4^{\circ}$ año de Educación Básica.

La idea de medir implica comparación. Por ejemplo, atendiendo a la longitud: más largo qué, más corto qué, más grande, más alto, más bajo, más chico. Luego, surge la necesidad de cuantificar, en que se buscar medir para asignar un valor a una longitud, superficie, volumen, etc. Antes de medir el contorno de superficies, se trabaja con longitudes continuas que pueden ser líneas rectas como curvas. Para calcular la longitud de una línea curva calculamos la longitud de una línea poligonal (varios segmentos de rectas unidos), que se obtiene sumando las longitudes de sus lados. En el contexto educativo podemos medir el perímetro de una línea curva con objetos físicos como hilo, lana, cinta de papel, etc., esto permite ajustes para después extenderlo longitudinalmente para su medición con una regla.

Se dice que la medida de la longitud de una curva cerrada plana es el perímetro de dicha curva que se medirá en unidades de longitud y se comienza el trabajo escolar con medidas de magnitud no estandarizas (cuarta, pie, lápiz, etc.) a estandarizadas (centímetros, metros, etc.). Desde el contexto educativo chileno, el currículo nacional define el perímetro como la medida del contorno de una superficie o de una figura y se expresa en unidades de longitud, por ejemplo, centímetros, metros, kilómetros. Para calcular el perímetro de un polígono se suman las medidas de sus lados.

Por otra parte, el área es la medida bidimensional de una región; es decir, se asigna un número real (un valor) y se acompaña de una unidad de medida (Fandiño y D' Amore, 2009). El área es una magnitud que expresa el tamaño o medida de una región y se mide en metros cuadrados, centímetros cuadrados, kilómetros cuadrados, etcétera, cuando empleamos medidas estandarizadas. Sin embargo, al igual que el perímetro es importante trabajar las unidades de magnitud de superficie empleando unidades que cubran la región plana y que no necesariamente sean unidades de medidas cuadradas o rectangulares. Cuando calculamos el área de una superficie debemos compararla con otra que elegimos como superficie unidad y averiguar el número de unidades que contiene; es decir, calcular el área de un cuadrado significa determinar cuántos cuadraditos o triángulos (por ejemplo) de una unidad de medida arbitraria cubren la superficie.

Es importante para el cálculo del área, trabajar los conceptos de congruencia y disección. En caso de las congruencias si tenemos dos regiones ( $R$ y $S$ ) congruentes entonces ambas regiones tienen la misma área $(\operatorname{rarea}(R)=$ área $(S))$. Para el caso de la disección una región $R$ se descompone en varias subregiones disjuntas, $A, B, \ldots, H$, entonces el área de $R$ es la 
suma de las áreas de las subregiones (área $(R)=$ área $(A)+$ área $(B)+\ldots+$ área $(H)$ ). Estos dos principios son fundamentales para trabajar áreas de figuras planas desde los primeros niveles escolares.

Desde un ámbito cognitivo, Montis, Mallocci y Polo (2003) confirman a través de la práctica, que los estudiantes entre $1^{\circ}$ a $4^{\circ}$ año de Educación Básica relacionan el área de las figuras según la medida del ancho o alto (según su percepción); es decir, aquella figura que tiene mayor medida de longitud de alto o de ancho, tiene mayor área. Olmo, Moreno y Gil (1993) plantean que la conservación del área y la diferencia existente con el perímetro son algunas de las dificultades que se encuentran en los estudiantes, debido a que a partir de que la superficie es sometida a determinadas transformaciones se produce el mismo trato para ambas medidas. Específicamente, los estudiantes no logran establecer la diferencia existente entre estas dos magnitudes.

Asimismo, el proceso de medida es complejo y hay que distinguir que una región se compone de subregiones y que pueden realizarse diferentes arreglos o reordenaciones para que no varíe su área; esta noción de disección (figura descompuesta en partes) implica desarrollar la visualización geométrica, para lo cual es necesario introducir los conceptos de la conservación del área, por tal motivo para el estudiante no variarían la cantidad de formas y/o espacios para poder medir la superficie. Por esto, se hace necesario que el alumno experimente las cualidades de la magnitud área al momento de adquirir los conceptos y antes de encontrarse con el procedimiento o la fórmula. Es fundamental que los alumnos lleguen a una necesidad de generar la formación del concepto de área diferenciada del perímetro.

Con respecto a la confusión entre perímetro y área, Olmo, Moreno y Gil (1993) manifiestan que es uno de los errores más frecuentes en los niños, y se produce generalmente cuando calculan perímetro y área de una figura, y asignan el dato mayor al área y el menor al perímetro. Entre otros errores más frecuentes, se cree que figuras con el mismo perímetro entonces tienen igual área. También los estudiantes se apropian del concepto de perímetro y área como magnitud con cualidades perceptibles que pueden ser medidas de manera arbitraria y estandarizada. En este sentido, cabe destacar la importancia de diseñar la unidad didáctica, ya que proporcionará a los alumnos un medio amplio para experimentar y verificar las experiencias en que se encuentre inmerso. Del mismo modo, se hace necesaria la existencia de tareas y/o actividades matemáticas en que se trabajen las distintas magnitudes y su medida en contextos o situaciones concretas.

En la secuenciación de dichas tareas y/o actividades es importante considerar las habilidades matemáticas que deben adquirir los alumnos, a través de las cuales se pretende establecer de manera clara y precisa, cuáles son los aprendizajes que deben lograr. De acuerdo con esto, el currículo nacional chileno define las habilidades como "capacidades de los estudiantes para realizar tareas y para solucionar problemas con precisión y adaptabilidad. Una habilidad puede desarrollarse en el ámbito intelectual, psicomotriz, afectivo y/o social" (Mineduc, 2012, p.12). De igual forma, se establecen cuatro habilidades, cuya importancia recae en el saber y el hacer, y que los estudiantes deben desarrollar en la asignatura de matemática: resolver problemas, modelar, representar, argumentar y comunicar. Estas habilidades serán consideradas para el diseño de las tareas matemáticas que conforman la unidad didáctica.

La actividad matemática centrada en una variedad de contextos es un aspecto importante para la alfabetización matemática que hoy la sociedad persigue. Es importante 
que los estudiantes se preparen para la vida, para lo cual es necesario razonar, analizar y comunicar operaciones matemáticas que lleven a utilizar el razonamiento matemático en la solución de problemas de la vida cotidiana. Los contextos son aquella parte del mundo del estudiante en los cuales se sitúan las tareas, con lo cual es importante que las tareas que se contextualizan en el aula contengan una variedad de contextos que permitan conectar intereses personales y situaciones en las que operan individuos del Siglo XXI (Watson y Callingham, 2003). En este trabajo consideramos los contextos de PISA (OCDE, 2014), que se contextualizan en cuatro aspectos para clasificar las tareas matemáticas: Personal, relacionadas al contexto inmediato de los alumnos y sus actividades diarias; Educativo o Laboral, relacionada con la escuela o el entorno de trabajo; Público, actividades centradas en la propia comunidad, ya sea local, nacional o global; y, Científico, implica el análisis de procesos tecnológicos o situaciones específicamente matemáticas. Estos contextos son la base para generar las tareas matemáticas de la secuencia de enseñanza sobre perímetro y área que contempla la unidad didáctica en este trabajo. Consideramos los cuatro contextos, ya que las pruebas de evaluación de conocimientos normalmente se guían por estos cuatro temas y engloban los contextos habituales de las actividades comunes que las personas realizamos.

Los procesos que el estudiante debe efectuar pueden tener tres grados de complejidad de las tareas matemáticas; según PISA (2012), se distinguen los siguientes aspectos que caracterizan las tareas matemáticas:

- Tarea de reproducción: Estas tareas se refieren a la reproducción del conocimiento a través de operaciones rutinarias de problemas y procedimientos matemáticos. Se consideran tareas simples en las que se emplean operaciones y cálculos comunes y/o simples, propios del entorno inmediato y la rutina cotidiana.

- Tarea de conexión: Exigen realizar interpretaciones y vincular diversas situaciones en contextos relativamente familiares. Se clasifican como tareas de conexión, aquellas cuya solución de problemas son de dificultad media. Igualmente, involucran ideas y procedimientos matemáticos para la solución de problemas, que ya no pueden definirse como ordinarios, pero que aun incluyen escenarios familiares.

- Tarea de reflexión: Estas tareas a menudo requieren razonamientos y conexiones matemáticamente complejas. Asimismo, pretende que los estudiantes identifiquen elementos que constituyen un problema matemático a través de la intuición y creatividad, que permitan desarrollar una aproximación a la matemática única. En último lugar, los alumnos deben matematizar o conceptualizar las situaciones, con el fin de adquirir y desarrollar en estos procesos un nivel mayor de abstracción.

Del mismo modo, las tareas matemáticas también deben atender a los conocimientos, habilidades y desempeño de los estudiantes al eje temático de medida en el caso de esta investigación y en los otros cuatro ejes temáticos, definidos por los programas de estudio de Matemática (Bases Curriculares Educación Básica, 2012).

La síntesis que resulta de este análisis muestra una planificación del aprendizaje, que inicia de la información del análisis de contenido, toma en consideración estos organizadores, y permite afrontar el diseño y selección de tareas que son los lineamientos para la construcción de la unidad didáctica sobre perímetro y área. 


\section{METODOLOGÍA}

La investigación es de carácter cualitativo, ya que la unidad didáctica diseñada e implementada permite obtener información específica de su diseño. Esto lleva a comprender si el material es adecuado para trabajar los temas matemáticos de perímetro y área para $4^{\circ}$ año de Educación Básica, con ajustes a la unidad didáctica para mejorar el proceso de enseñanza y aprendizaje.

Las investigaciones situadas en el paradigma cualitativo, en el contexto educativo, buscan entregar soluciones a problemas. Este tipo de investigación se caracteriza por ser aplicada en contextos sociales, de modo de entender y mejorar los fenómenos en profundidad. Además, este estudio es una investigación-acción, ya que como la define Cohen, Manion y Morrison (2011) la intervención es a pequeña escala en el funcionamiento del mundo real y un examen próximo de los efectos de tal intervención, por tanto, al implementar la unidad didáctica se pretende evaluar, reflexionar y reportar resultados concretos de la ejecución de la unidad. Siendo una investigación acción participativa, las investigadoras de este trabajo son partícipes directamente del diseño, implementación y evaluación de la unidad didáctica, con el objetivo de mejorar la práctica y el proceso de aprendizaje de sus estudiantes.

Cabe mencionar, que la investigación-acción se lleva a cabo a partir de un proceso cíclico; es decir, una vez que finaliza vuelve a comenzar, y contempla fases de planificación, observación, acción y reflexión. Además, existe una relación entre la fundamentación teórica y práctica, por lo tanto, la teoría obedece a la práctica y viceversa. El o los investigadores tienen un rol fundamental, ya que son quienes deben acercarse lo más posible al fenómeno de estudio, con el fin de comprender, explicar e interpretar detalladamente el significado de la problemática.

De acuerdo con el planteamiento del problema identificado en esta investigación, abordamos el diseño y evaluación de la unidad didáctica según las fases del análisis de evaluación. En este sentido, se realizan tres fases fundamentales para el diseño, implementación y evaluación de la unidad, que describimos a continuación.

\section{DISEÑO DE LA UNIDAD DIDÁCTICA}

Primeramente, el diseño de la unidad didáctica para $4^{\circ}$ año de Educación Básica sobre los conceptos de perímetro y área contempla los temas matemáticos desarrollados en el análisis conceptual y en el análisis de contenido. A su vez, considera los distintos errores y dificultades asociados a los temas matemáticos que aborda la unidad didáctica. Además, la unidad didáctica se secuencia según una variedad de tareas matemáticas, atendiendo a los Objetivos de Aprendizaje para el nivel escolar. Conjuntamente busca desarrollar las habilidades matemáticas propuestas por el Ministerio de Educación (2012); los contextos de PISA (2012) como el científico, personal, educativo o laboral y público; los grados de complejidad de las tareas matemáticas, como tareas de reproducción, conexión y reflexión; y una variedad de sistemas de representación (simbólico/verbal/figural/ manipulativo) para trabajar los temas de perímetro y área.

Para la secuenciación de la unidad didáctica se construyó una matriz de análisis cualitativa, en la cual se consideran cinco categorías: objetivo de aprendizaje, habilidades matemáticas, contextos, niveles de complejidad de las tareas y sistemas de representación, 
con sus respectivas sub-categorías. Los objetivos de aprendizaje (OA) que guían el diseño de la unidad didáctica corresponden a aquellos definidos para $4^{\circ}$ año de Educación Básica; es decir el OA 23. Este objetivo es desglosado en cinco objetivos parciales para plantear tareas específicas que atiendan al desarrollo del OA 23. La siguiente categoría denominada Habilidades Matemáticas, implica la capacidad de un individuo de identificar y entender el papel que las matemáticas tienen en el mundo. En este sentido, se consideran las siguientes habilidades: Resolver problemas; Modelar; Representar; y Argumentar y Comunicar.

La categoría Contextos, alude a que los problemas matemáticos planteados están situados en los diferentes contextos o también llamados situaciones, para ello definimos los contextos empleados por PISA (2012) y las categorías adoptadas en este trabajo: personal, educativo o laboral, público y científico. La categoría sobre los niveles de complejidad de las tareas matemáticas, corresponden a tres grados de complejidad que se busca desarrollar en las tareas de la unidad didáctica: reproducción, conexión y reflexión. Por último, la categoría sistema de representación considera cuatro registros de representación que son desarrollados a partir de las tareas matemáticas de la unidad didáctica: simbólico, verbal, figural y manipulativo.

En síntesis, para cada objetivo de aprendizaje descrito se considera una variedad de tareas matemáticas ajustadas a los contextos, niveles de complejidad de las tareas y variedad de sistemas de representaciones, que busquen desarrollar las habilidades que el currículo nacional lleva a desarrollar en los estudiantes. La matriz quedó conformada por 31 tareas matemáticas en contextos regionales (plaza, estadio regional, artesanía de la Segunda Región, fiestas patrias, entre otros), graduadas con distinta complejidad; es decir, se reformularon tareas de reproducción con distinta complejidad, Además, las tareas pretendían ser abordadas con materiales o recursos acordes para el nivel escolar. Las 31 tareas fueron secuenciadas de acuerdo con el objetivo de aprendizaje y en ocasiones se reformularon para potenciar los indicadores de cada tarea. También se buscó un equilibrio en cuanto al número de tareas para cada OA y una proporción de aspectos de cada categoría. Las tareas que son parte de la unidad didáctica fueron analizadas en su contexto, representaciones, nivel de complejidad y habilidades matemáticas a desarrollar. Se realizó, además, para cada tarea la respuesta matemática o respuesta experta y dos posibles estrategias de resolución que los estudiantes podrían emplear en su desarrollo.

Para la implementación se consideran 26 tareas matemáticas, y algunas no se implementaron debido a que atendían al mismo objetivo y otras eran de reproducción. Las tareas implementadas fueron organizadas en cinco guías de trabajo y otras empleadas en la ejecución directa de la clase, para activar conocimientos previos al inicio de la clase. En la Figura 1 presentamos un modelo de guía desarrollada para la implementación de la unidad didáctica.

Las guías se contemplaron en las planificaciones de clase en que se ejecutó la unidad didáctica. Además, para el análisis se elaboró una lista de cotejo para examinar el alcance los objetivos de aprendizaje.

Considerando que Gurdían-Fernández (2007) propone que es necesario que el investigador utilice estrategias de verificación durante el proceso de investigación, previamente a la implementación de la unidad didáctica, esta fue analizada y evaluada por seis profesores en el área de las matemáticas, lo que le dio validez al material diseñado. Los señalamientos fueron fundamentalmente para mejorar los enunciados de las tareas y algunas preguntas de las situaciones. 


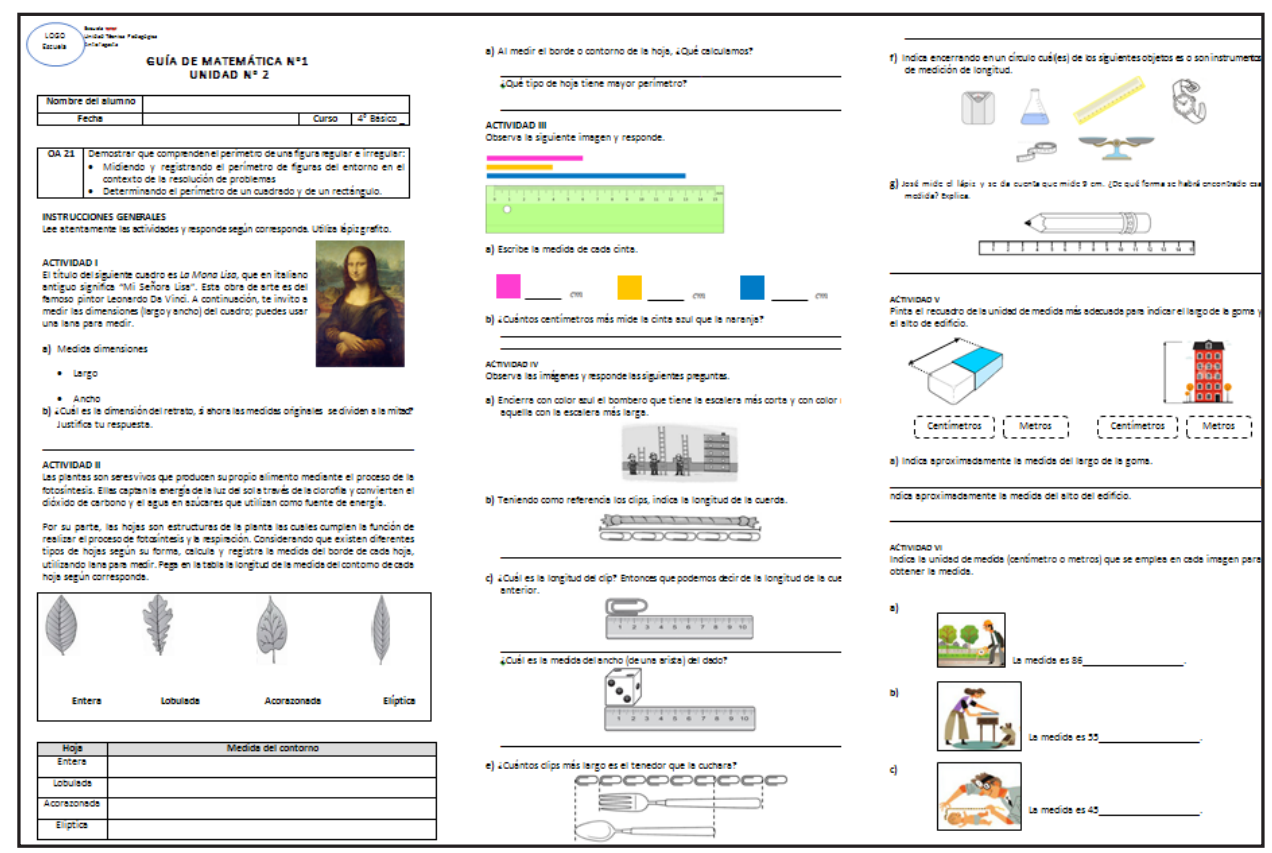

Figura 1. Guía №1 Unidad Didáctica

\section{IMPLEMENTACIÓN DE LA UNIDAD DIDÁCTICA}

Para la implementación de la unidad didáctica seleccionamos un curso de $4^{\circ}$ año de Educación Básica, ya que como se mencionó en el análisis curricular las habilidades de medir longitudes y superficies se comienzan a estudiar en cuarto año. Desde $3^{\circ}$ año de Educación Básica se trabaja el tema de perímetro y en $4^{\circ}$ año hasta $6^{\circ}$ año el cálculo de superficies. Asimismo, en este nivel escolar se hace la transición de trabajar magnitudes de longitud a magnitudes de área; en este nivel la unidad didáctica implementada busca que los estudiantes diferencien y comprendan estos conceptos.

El establecimiento educacional y el curso se seleccionan por disponibilidad; es decir, se consulta en una escuela municipal la posibilidad de aplicar la unidad didáctica, y su director accede a facilitar la intervención. Hay que indicar que el índice de vulnerabilidad de la escuela es de $69,1 \%$, por lo cual es un establecimiento idóneo para aportar en sus aulas.

La unidad didáctica se implementó en un $4^{\circ}$ año de Educación Básica de una Escuela Municipal de la Región de Antofagasta. El curso tiene una matrícula de 32 estudiantes de nivel socioeconómico bajo y promedio general 5.4 en la asignatura de matemática (en escala de 1 a 7). Cinco estudiantes del curso en que se produce la intervención pertenecen al Proyecto de Integración Escolar (PIE) y ellos también fueron participantes de la implementación de la unidad didáctica. La unidad didáctica se llevó a cabo en el horario habitual de la asignatura (tres veces a la semana), y se realizó durante 11 clases de 90 minutos cada una, correspondientes a 22 horas pedagógicas de intervención en el aula. 
Cada clase fue realizada por una de las investigadoras de este estudio, que tenía previstas las respuestas matemáticas de cada situación. Además, el profesor titular del curso observó cada clase y comentó a las profesoras-investigadoras algunas apreciaciones sobre la implementación de cada clase. Las clases fueron grabadas en audio, con el consentimiento de los apoderados y profesor titular de la asignatura. En este estudio se recurre al audio solo en caso de ser necesario; es decir, por no tener claridad del avance de los estudiantes frente a la tarea o para revisar los indicadores de logro en las listas de cotejo cuando las producciones de los estudiantes no son claras.

\section{EVALUACIÓN DE LA UNIDAD DIDÁCTICA}

La unidad didáctica fue evaluada de acuerdo con el análisis evaluativo. Este análisis

utiliza la información que surge de la puesta en práctica de las actividades de enseñanza y aprendizaje para producir información que permita determinar la comprensión de los escolares en ese momento, los contenidos a tratar en el aula y los objetivos de aprendizaje que se deben buscar en el nuevo ciclo (Gómez, 2007, pp. 93-94).

Esta fase contempló una revisión del proceso de implementación de la unidad didáctica diseñada, con el fin de analizar el diseño y modificarlo.

Las fases a priori contempladas en el análisis de evaluación es el análisis de contenido, que permitió organizar los significados relacionados con los temas de perímetro y área, los sistemas de representación, análisis fenomenológico y estructura conceptual; asimismo, se incluye un análisis curricular, en el cual se visualiza en qué niveles de escolaridad los estudiantes inician el aprendizaje de los conceptos de perímetro y área. En segundo lugar, contempla el análisis cognitivo en el cual se describen las competencias matemáticas que los estudiantes deben desarrollar en el proceso de enseñanza y aprendizaje de dichos temas matemáticos. En atención a ello, inicia una nueva fase, el análisis de instrucción, en el que se propone una secuencia de actividades de enseñanza y aprendizaje para perímetro y área, considerando los contextos de los estudiantes y el nivel de complejidad de las tareas matemáticas.

Concretamente se comparan las previsiones que se hicieron en la planificación con aquello que sucede cuando esa planificación se pone en práctica en el aula, y se establecen los logros y deficiencias de la planificación (actividades y tareas) en su puesta en práctica. Además, se reflexiona sobre el aprendizaje de los escolares producto de la propuesta de enseñanza implementada, lo que genera información relevante para ajustar la planificación y mejorar el material diseñado. En este sentido, el análisis de evaluación, en esta investigación es un análisis a posteriori, el cual se focaliza en la valoración del material diseñado según los aspectos mencionados, y en el cual se evalúan fortalezas y debilidades de la unidad didáctica diseñada e implementada. Cabe destacar que los análisis y reflexiones realizados previamente son fundamentales para esta fase, ya que es una consecución de la investigación.

La evaluación de la unidad didáctica se realiza según cada tarea matemática, considerando los porcentajes de logro de la tarea. Además, se realiza un análisis general de cada guía aplicada en el aula, con el objeto de tener una panorámica clase a clase de los avances adquiridos por los estudiantes. Luego, se efectúa un análisis de la unidad global, 
por tareas aplicadas en todas las clases, para identificar las fortalezas y debilidades de la unidad didáctica implementada.

\section{RESULTADOS}

En esta investigación evaluamos la unidad didáctica según el material diseñado, correspondiente a las guías secuenciadas según tareas matemáticas de diversos niveles de complejidad, variados contextos y relacionados con los objetivos de aprendizaje establecidos por el Misterio de Educación chileno para $4^{\circ}$ año de Educación Básica. El proceso de evaluación beneficia tanto a los docentes como a los estudiantes para conocer los avances de los contenidos y áreas que se necesitan fortalecer, y para dar continuidad al proceso de enseñanza y aprendizaje, además se pueden tomar decisiones para adecuar la planificación.

En lo que sigue, presentamos el análisis de dos tareas matemáticas de la Guía $\mathrm{N}^{\circ} 2$ que tenía por objetivo demostrar que se comprende el concepto de perímetro, para lo cual se mide y se registra el perímetro de figuras regulares (cuadrados y rectángulos) con el empleo de unidades de medida estandarizadas y operaciones matemáticas básicas.

Tarea matemática 1 (Figura 2), relacionada con el objetivo de aprendizaje Midiendo y registrando el perímetro de figuras del entorno en el contexto de la resolución de problemas, permite desarrollar diversas habilidades como representar, modelar, resolver problemas, y argumentar y comunicar. La tarea matemática está dada en un contexto social, su complejidad es de conexión, ya que para su resolución moviliza conocimientos sobre adición y sustracción de números naturales, división, múltiplos, divisores y figuras planas. Además, permite trabajar con representaciones verbal, simbólica, figura y material.

El título del siguiente cuadro es La Mona Lisa, que en italiano antiguo significa

"Mi Señora Lisa". Esta obra de arte es del famoso pintor Leonardo Da Vinci. A continuación, te invito a medir las dimensiones (largo y ancho) del cuadro; puedes usar una lana para medir.

b) ¿Cuál es la dimensión del retrato, si ahora las medidas originales se dividen a la mitad? Justifica tu respuesta.

a) Medida dimensiones: largo y ancho.

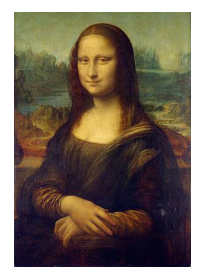

Figura 2. Tarea matemática 1

La tarea matemática se relaciona con cinco indicadores de evaluación, tal como se indica en la Figura 3. Los tres primeros indicadores fueron logrados por la totalidad de los estudiantes que asistieron a clases (23 alumnos), estos se orientan a medir y registrar con lana (trabajo con medida no estandarizada) el largo y el ancho de una figura, con el propósito de que los estudiantes construyan la idea de medida de longitud. A partir de la ejecución de la tarea se reconoce que el rectángulo tiene dos pares de medidas de igual longitud. 


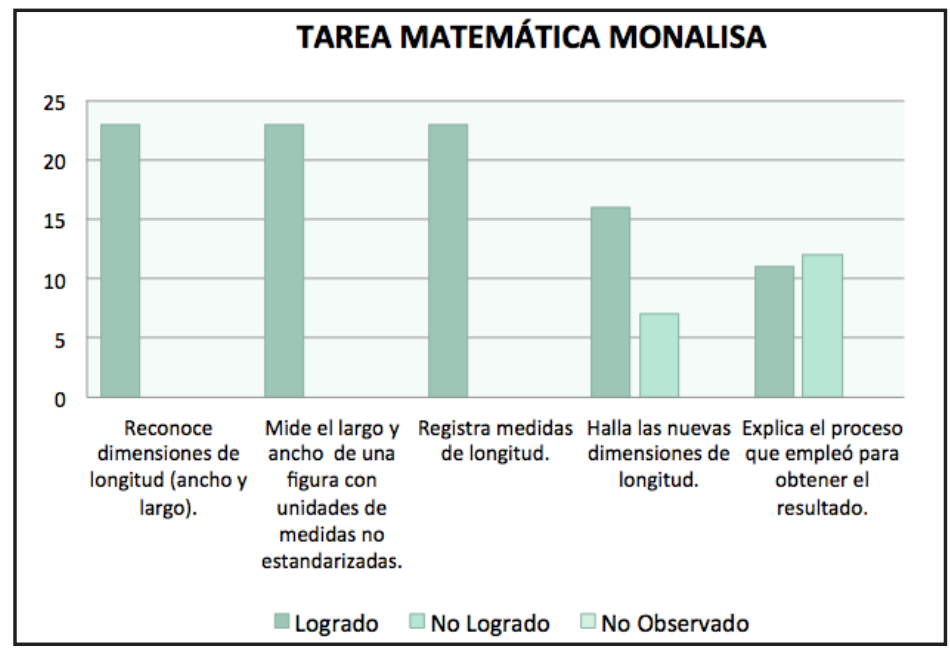

Figura 3. Tarea matemática 1

Al trabajar iteraciones, dividiendo una figura a la mitad, para que los estudiantes comprendieran que la longitud disminuía al volver a dividirla, esto presentó dificultad para los estudiantes. Producto de que la tarea tenía dos posibilidades de resolución: dividir el ancho y el largo a la mitad de longitud o bien dividir la figura a la mitad, disminuyendo el largo y fijando el ancho o viceversa, como se observa en la Figura 4.

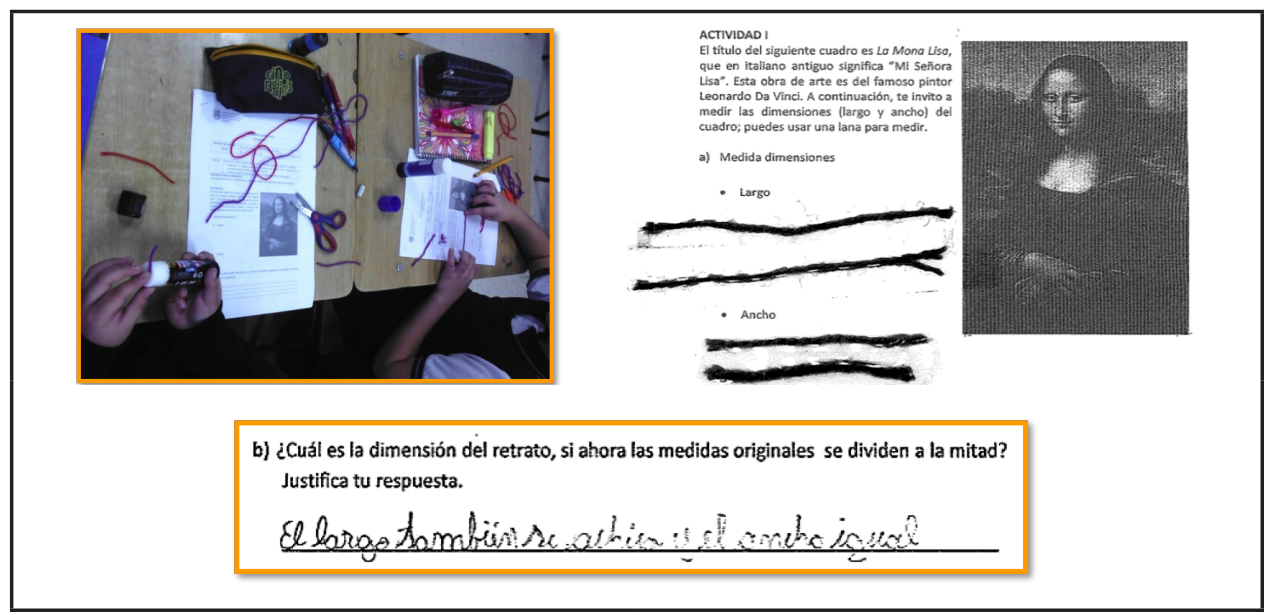

Figura 4. Estudiantes midiendo y registrando el largo y ancho con unidades de medida no estandarizadas. 
El análisis de la tarea permite realizar un ajuste al enunciado de la pregunta para que sea más explícito en función a dividir el largo y el ancho del retrato a la mitad. La mayoría de los estudiantes no logran argumentar y/o comunicar de forma escrita el proceso que emplearon para desarrollar la actividad; sin embargo, argumentan a partir de la representación verbal. Considerando que esta habilidad es transversal, se sigue desarrollando en las restantes tareas matemáticas.

Se analiza la tarea 11 (Figura 5) cuyo objetivo es medir y registrar la medida de superficie de dos figuras irregulares empleando medida no estandarizada, para ello se usan lentejas. La tarea, que permite trabajar habilidades de representación, modelar, y argumentar y comunicar resultados matemáticos, está en un contexto personal en el cual los estudiantes deben contar objetos. La tarea es de reproducción al contar una cantidad de elementos de una colección y permite el paso de representaciones manipulativa, verbal y simbólica.

Mide las siguientes superficies empleando legumbres.

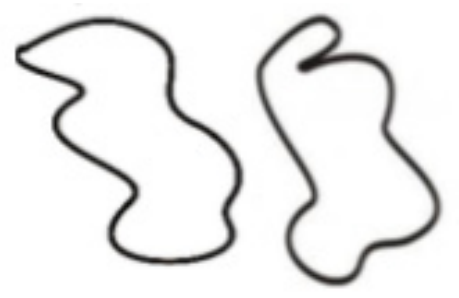

a) ¿Qué figura ocupa mayor superficie?, ¿Por qué? y ¿Cuál es su medida?

Figura 5. Tarea matemática 25

El 100\% de los estudiantes (un total de 23 alumnos) logró medir y registrar las medidas de superficie de las figuras irregulares empleando unidades de medida no estandarizadas en la tarea. Sin embargo, al comparar las medidas de superficie de ambas figuras un $3 \%$ no logró hacerlo correctamente. Cabe destacar que, para obtener la medida de superficie de las figuras, los estudiantes contaron la cantidad de lentejas. Durante el desarrollo de la clase se observó que algunos alumnos presentaron dificultad en el conteo. Además, el 10\% no logró verbalizar y/o comunicar explícitamente su respuesta matemática.

Esta tarea matemática fue motivadora para los estudiantes, ya que el empleo de material concreto fue una experiencia enriquecedora que favoreció el aprendizaje significativo y metódico, con el desarrollo del pensamiento matemático, lógico y crítico. Con el análisis se ha decidido no realizar ajustes a esta tarea matemática, solo se considerarán adecuaciones respecto al formato; es decir, los enunciados serán explícitos y se establecerán los espacios necesarios para las posibles respuestas. El análisis se efectúa a 25 tareas matemáticas respecto a indicadores que se relacionan con los objetivos de aprendizaje, con lo cual se establecen las siguientes conclusiones. 


\section{CONCLUSIONES}

Valorando el objetivo de implementación de la unidad didáctica en un cuso de cuarto año de Educación Básica, podemos indicar que la secuenciación de las tareas matemáticas y su implementación permitieron ajustar la unidad en relación con los aspectos indicados en la Tabla 1.

Tabla 1. Ajustes realizados a la unidad didáctica

\begin{tabular}{|c|c|}
\hline Ajustes a la unidad & $\mathbf{N}^{\circ}$ de Tareas \\
\hline Enunciado & $7 / 25$ \\
\hline Formato & $7 / 25$ \\
\hline Representación & $1 / 25$ \\
\hline Contexto & $1 / 25$ \\
\hline Secuenciación & $6 / 25$ \\
\hline Sin ajustes & $9 / 25$ \\
\hline
\end{tabular}

De un total de 25 tareas evaluadas en la unidad didáctica, nueve no requirieron ajuste, debido a que se relacionaban con los objetivos de aprendizaje, con un alto porcentaje de logro de estas tareas. Los mayores ajustes de las tareas fueron en el enunciado y formato, en los cuales se cambió el lenguaje con términos más personales para los estudiantes, y se explicitaron las indicaciones de las tareas en cada subtarea, para que no surgieran ambigüedades en las indicaciones para abordar la situación.

El formato de siete tareas fue modificado, principalmente los espacios preestablecidos para responder las tareas como argumentar, realizar desarrollos de operaciones, dibujar, entre otras. Esto se debe a que los estudiantes están en una etapa concreta en la cual las indicaciones deben ser metódicas y fáciles de comprender.

De un total de 25 tareas diseñadas solo una de ellas tuvo ajuste en el contexto. Esto, debido a que la unidad de medida de longitud como el centímetro y el metro no eran dimensionadas por los estudiantes. Es decir, son capaces de hacer la conversión de centímetros a metros y viceversa; sin embargo, no establecen comparación entre objetos con estas unidades de medidas. La tarea que cambia en contexto, también se cambia en el sistema de representación empleado, para llevarla a un contexto personal en que la disparidad de medidas es más evidente para los alumnos.

De las tareas consideradas en la unidad didáctica seis se cambiaron de orden, esto producto de que estaban relacionadas con otras tareas planteadas, además permiten profundizar en los temas matemáticos relacionados con otros objetivos de aprendizajes. Solo una tarea matemática fue eliminada de la unidad didáctica, por tener un nivel de complejidad alto, más pertinente para un nivel escolar mayor. La tarea eliminada de la unidad empleaba conceptos más abstractos para su resolución, principalmente división de tres cifras en el dividendo, tarea más apropiada para $5^{\circ}$ o $6^{\circ}$ año, cuando tienen automatizada la división (Bases Curriculares, 2012). 
La unidad didáctica buscaba que los estudiantes construyeran su propio significado referente a los temas de perímetro y área, a través del desarrollo de tareas matemáticas contextualizadas, que presentaban situaciones desafiantes que requerían poner en juego habilidades, destrezas y conocimientos, no necesariamente con la aplicación de fórmulas o algoritmos que los condujeran a los resultados de forma procedimental. Respecto al comportamiento de la unidad didáctica a lo largo del proceso de implementación y, posteriormente, en su evaluación se evidencia un alto porcentaje de logro de las tareas por parte de los estudiantes asistentes a las clases, tal como lo indica la Figura 6.

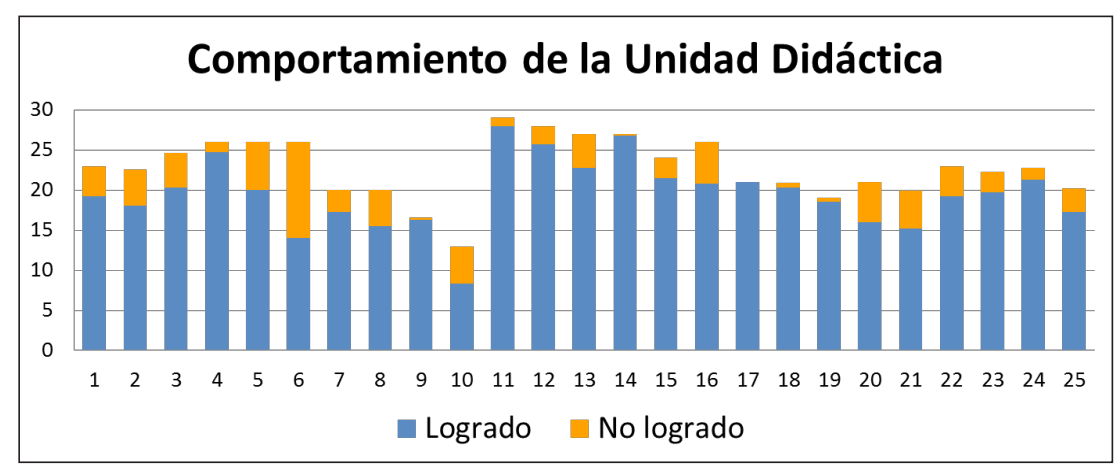

Figura 6. Comportamiento de la Unidad Didáctica

Específicamente, identificamos un mayor porcentaje de no logro en las tareas matemáticas orientadas al proceso de enseñanza y aprendizaje del perímetro (tareas matemáticas desde la 1 a la 10), aunque menos de 10 alumnos eran los que presentaron dificultades con el contenido. Por ejemplo, los mayores errores y dificultades se presentaron en la tarea matemática 6 , la cual se relacionaba con establecer unidades de medida estandarizadas en contextos reales. Sin embargo, eran capaces de demostrar que el metro es la unidad de medida básica del Sistema Internacional y que el centímetro es una unidad que deriva de él; también reconocían equivalencias o transformación de unidades de medidas, particularmente cuántos centímetros equivalen a un metro y viceversa. No obstante, era complejo visualizar y distinguir cuál de ellas se utiliza para medir elementos con un largo mayor o menor en contextos cercanos. Esto permitió organizar en la unidad didáctica, las tareas en contextos personales y sociales y vincularlas con el tema de perímetro. Como mencionan Fandiño y D' Amore (2009) es necesario trabajar las unidades de magnitud de superficie empleando unidades que cubran la región plana y que no necesariamente sean unidades de medidas cuadradas o rectangulares, aspectos que se pueden abordar en una variedad de contextos no matemáticos.

Podemos indicar que, si bien el significado de perímetro fue complejo de construir por parte de los estudiantes, sí se logró que ellos comprendieran el paso de una medida de longitud a una de área. Así, se logró que los alumnos no relacionaran el perímetro y área como magnitudes equivalentes; como indica Corberán (1996) es común que los estudiantes piensen que el área y el perímetro son medidas de superficies, concepción 
errónea que les impide ver el área como una propiedad de la superficie independiente del perímetro.

Las tareas matemáticas correspondientes al proceso de enseñanza y aprendizaje del concepto de área (tareas matemáticas de la 11 a la 25) tuvieron un mayor porcentaje de logro. El área se concibió como una medida de superficie, en la cual se puede emplear cualquier medida para estimarla (lenteja, triángulo, cuadrado de distintas medidas, entre otros). Estas ideas son clave en el proceso de adquisición de la medida, que es una noción abstracta que se aleja del saber que muchas veces se trabaja en la escuela (medir solo con un instrumento), y se establece una comparación con referentes fijos, que implica cuántas veces "algo" (una unidad) está contenida en el objeto a medir.

Por otra parte, de la evaluación de la unidad didáctica se desprenden debilidades y fortalezas. Por ejemplo, una de las debilidades de la unidad didáctica fue su extensión: se planificaron seis clases, en las que se consideraron cinco guías de proceso y una evaluación final de la unidad. Sin embargo, fue complejo para los estudiantes realizar una guía de trabajo por clase, y tuvieron que desarrollarla en más de una sesión. Debido a ello, se realizaron los ajustes y en las guías de proceso se consideraron menos tareas matemáticas, con el objetivo de que los estudiantes tuviesen más tiempo para resolver las tareas. Otra debilidad fue el lenguaje empleado en las tareas, ya que en ocasiones las indicaciones no eran comprendidas por los estudiantes por desconocer algunas palabras, lo cual llevó a emplear un lenguaje más cercano a los estudiantes con frases cortas y precisas. Para ello, hubiese sido interesante y pertinente presentar previamente las tareas a un grupo de niños para consultar si se entienden las indicaciones.

Los aspectos positivos de la unidad didáctica fueron varios, como se ha ido indicando. El diseño de unidades didácticas es un instrumento potencial para profundizar en las problemáticas educativas $\mathrm{y}$, fundamentalmente, en los errores y dificultades que presentan los estudiantes al estudiar conceptos matemáticos de diversos ejes temáticos en todos los niveles educativos. Secuenciar las tareas matemáticas respecto a los objetivos de aprendizaje que el currículo nacional exige, además, busca desarrollar habilidades en los estudiantes. Este es un ejercicio que en la práctica se aborda de forma exigua, por lo cual es necesario planificar la actividad matemática del aula en relación con las exigencias nacionales e internacionales, así como con las necesidades de los estudiantes.

Igualmente, esta investigación es relevante para el desarrollo de competencias y aprendizajes no solo de estudiantes, sino que también de la labor docente de profesores, mediante la creación y diseño de diversas actividades secuenciadas para el nivel escolar, proceso cíclico que permitió una reflexión profunda acerca del trabajo del profesor. Como mencionan Cohen, Manion y Morrison (2011) el valor particular de la investigación científica en la educación es tal que capacitará a los educadores para desarrollar la clase de conocimientos sólida que caracteriza a otras profesiones y disciplinas, y que le asegura a la educación una madurez y sentido de progreso del que carece en el presente, con lo cual esta investigación busca ser un aporte al desarrollo profesional docente.

Considerando que la unidad didáctica tenía por objetivo que los estudiantes comprendieran los conceptos de perímetro y área (más que la mecanización), mediante la resolución de tareas matemáticas en contextos de interés para ellos y con niveles progresivos de abstracción mayor, el proceso metodológico seguido para el diseño de la unidad didáctica se presenta como una buena herramienta para generar propuestas de enseñanza en matemáticas acorde con los estándares internacionales. 


\section{REFERENCIAS BIBLIOGRÁFICAS}

Cohen, L., Manion, L. y Morrison, K. (2011). Research methods in education. London: RoutledgeFalmer.

Corberán, R. M. (1996). Análisis del concepto de área de superficies planas. Estudio de su comprensión por los estudiantes desde primaria a la Universidad (Tesis de Doctorado). Universidad de Valencia, España.

Espinoza, L., Barbé, J. y Gálvez. G. (2011). Limitaciones en el desarrollo de la actividad matemática. Estudios Pedagógicos, 37(1), 105-125.

Fandiño M. y D’Amore B. (2009). Área y perímetro. Aspectos conceptuales y didácticos. Bogotá: Magisterio.

Gómez, P. (2007). Desarrollo del conocimiento didáctico en un plan de formación de profesores de matemáticas de secundaria (Tesis doctoral). Universidad de Granada, España.

Gurdían-Fernández, A. (2007). El paradigma cualitativo en la investigación socio-educativa. San José, Costa Rica: Colección IDER.

Ministerio de Educación de Chile (2009). Ley General de Educación (LGE), de 17 de agosto, ISN CHL-2009-L-82695.

Ministerio de Educación (2012). Bases Curriculares. Chile: Gobierno de Chile.

Montis, A., Mallocci, P. y Polo, M. (2003). Congettura e argomentazione nella construzione dei concetti di equiestensione e isoperimetria: un percorso didattico dalla prima alla quinta elementare. L'educazione Matemática, 5(1), 1-12.

OCDE (2014). PISA 2012 Results: What Students Know and Can Do. Student Performance in Mathematics, Reading and Science (Vol. 1), PISA, OECD Publishing. doi: 10.1787/9789264201118-en

Olmo, M., Moreno, M. y Gil, F. (1993). Superficie y volumen ¿algo más que el trabajo con fórmulas. Madrid: Editorial Síntesis.

Rico, L. (2013). El método del análisis didáctico. Revista Iberoamericana de Educación, 33, 11-27.

Rico, L., Lupiáñez, J. y Molina, M. (2013). Análisis Didáctico en Educación Matemática: Metodología de investigación, formación de profesores e innovación curricular. Granada, España: Universidad de Granada.

Watson, J. y Callingham, R. (2003). Statistical literacy: A complex hierarchical construct. Statistics Education Research Journal, 2(2), 3-46. 
